04

\title{
Исследование механизма записи мультиплексных брэгговских дифракционных решеток с планарным вводом-выводом оптического излучения в стеклянных световодах
}

\author{
(C) С.Б. Одиноков, М.В. Шишова, А.Ю. Жердев, Д.С. Лушников, В.В. Маркин \\ Московский государственный технический университет им. Н.Э. Баумана, \\ Москва, Россия \\ e-mail: odinokov@bmstu.ru
}

Поступила в редакцию 15.12 .2020 г.

В окончательной редакции 15.12.2020 г.

Принята к публикации 22.12.2020 г.

Описана запись мультиплексных брэгговских дифракционных решеток для световодных дисплеев по методу оптического копирования с применением фазовой маски. Световоды в данном эксперименте были изготовлены из фото-термо-рефрактивного стекла. В качестве фазовой маски использовалась фоторезистивная рельефно-фазовая дифракционная решетка, нанесенная на световодную пластину. На основе исследований по угловому мультиплексированию реализован компактный дисплей дополненной реальности.

Ключевые слова: дифракционные, брэгговские решетки, мультиплексирование, голографическая литография, фазовые маски, дифракционные волноводы, дисплей дополненной реальности.

DOI: $10.21883 /$ OS.2021.04.50770.297-20

\section{Введение}

Развитие дисплеев дополненной реальности $[1,2]$ и устройств интегральной фотоники [3] поставило перед исследователями задачу разработки новых методов записи волноводных голограмм и создания дифракционных световодов. В таких систах дифракция света используется для введения излучения в стеклянную пластину, где излучение распространяется с помощью полного внутреннего отражения (ПВО). Дифракционные решетки в роли вводных и выводных ответвителей для дисплеев дополненной реальности могут быть как рельефно-фазовыми, так и объемными фазовыми, но угол дифракции внутри подложки должен удовлетворять условию ПВО. Запись волноводных дифракционных элементов связана с возрастанием инженерной сложности экспериментальных стендов записи, так как необходимо обеспечить сравнительно высокие углы сведения записывающих пучков: при записи объектная волна должна находиться внутри подложки. Применение объемных голографических решеток в контексте устройств дополненной реальности позволяет увеличить энергетическую эффективность системы [4,5]. Объемные голографические решетки имеют высокую угловую и спектральную селективности, поэтому для реализации дисплея на их основе требуются голографические материалы с высокой модуляцией показателя преломления, и/или пространственное мультиплексирование. Работа демонстрирует стабильный метод обеспечения описанных условий. Создание мультиплексированных брэгговских решеток для световодов из фото-термо-рефрактивного (ФТР) стекла является целью исследования.

ФТР стекло в качестве голографической объемной среды уникально для интеграции фазовых дифракцион- ных элементов с волноводной платформой [6] и обладает высокой стойкостью к внешним воздействиям (температуре, влажности и давлению) и химической стойкостью [7]. Фазовая структура формируется непосредственно внутри среды, поэтому нет необходимости защищать световод дополнительным стеклом. Стеклянная подложка представляет собой голографический материал, поэтому мы можем записывать голограммы по всей толщине подложки. Неоспоримым преимуществом ФТР стекла для голографии является отсутствие усадки. Тогда при соблюдении технологического процесса записанная структура формируется без искажений и в высокой степени соответствует расчетным параметрам.

Метод записи с применением фазовой маски реализует задачу оптического копирования [8] рельефно-фазовой дифракционной решетки в объемную среду и создания брэгговской дифракционной решетки. Он не требует значительной виброизоляции из-за отсутствия интерферометрических ветвей в оптической схеме [3,9-12]. Представленное исследование отличается наклонным освещением фазовой маски одиночным регистрирующим лазерным пучком, что позволяет изготавливать наклонные брэгговские решетки. Рельефно-фазовая дифракционная решетка выбрана в качестве фазовой маски для удобства углового мультиплексирования, так как она неселективна [13].

\section{1. Принципы записи}

Запись с использованием фазовой маски обладает рядом преимуществ по сравнению со стандартной регистрацией интерференционной картины, когда реализован 
интерферометр с делением по амплитуде. Этот тезис подтверждают следующие аргументы.

1. Для обеспечения высоких частот брэгговских решеток требуются высокие углы сведения записывающих пучков. В предложенном методе это реализуется с помощью дифракционной решетки в качестве фазовой маски, углы записи представляют собой углы дифракции.

2. Плоский равномерный волновой фронт должен быть создан только для одной ветви. В описанном эксперименте объемные решетки изготавливаются в широком пучке диаметром не менее $40 \mathrm{~mm}$. Запись всей площади дифракционного световода регистрируется одновременно. Создание однородного плоского волнового фронта такого размера становится нетривиальной задачей для ультрафиолетового излучения, которое актинично для ФТР стекла.

3. Требования к виброустойчивости установки записи снижены. Поскольку образец и шаблон объединены в единый элемент, механические колебания возникают относительно „сэндвича“ фазовой маски и ФТР подложки, и интерференционная картина не смещается во время записи из-за вибраций. Для сравнения, при получении голографических дифракционных решеток вибрации не должны превышать погрешность изготовления периода дифракционной решетки. Этот момент выгоден для продолжительных экспозиций.

\section{1. Особенности фото-термо-рефрактивного стекла}

ФТР стекла имеют сложный химический состав $\mathrm{Na}_{2} \mathrm{O}$ $\mathrm{ZnO}-\mathrm{Al}_{2} \mathrm{O}_{3}-\mathrm{SiO}_{2}-\mathrm{NaF}$. Для создания фоточувствительности их активируют оксидами серебра $\mathrm{Ag}_{2} \mathrm{O}$, церия $\mathrm{Ce}_{2} \mathrm{O}_{3}$, олова $\mathrm{Sn}_{2} \mathrm{O}_{3}$. Каждый функциональный компонент участвует в формировании особых свойств материала. Для формирования фоточувствительности в ультрафиолетовой области спектра излучения в состав ФТР стекла включают оксиды церия $\mathrm{Ce}_{2} \mathrm{O}_{3}$. Тогда после облучения УФ светом в полосе поглощения ионов трехвалентного церия Се(III) происходит фотоионизация ионов церия с образованием свободных электронов. Таким образом, спектральный диапазон фоточувствительности ФТР стекла обусловлен шириной полосы поглощения церия и лежит в области 290-340 nm с пиком в области 305-310 nm. В представленном исследовании регистрация интерференционного поля проводилась с помощью Не-Cd-лазера с длиной волны $325 \mathrm{~nm}$. При постобработке ФТР стекло нагревают. Изменение показателя преломления происходит за счет создания центров фотоионизации при взаимодействии фотонов с ионами сурьмы, частично ионами серебра, анионными вакансиями в стекле и примесными центрами с образованием различных молекулярных кластеров. Дальнейшее увеличение температуры до температуры стеклования $\left(\approx 500^{\circ} \mathrm{C}\right)$ приводит к агломерации молекулярных кластеров с образованием коллоидной наночастицы серебра и далее к образованию металлической наночастицы, затем к росту наноразмерной кристаллической оболочки из бромида серебра и бромида натрия. Термообработка при температурах, близких или больших температуры стеклования, приводит к росту на оболочке $\mathrm{AgBr}-\mathrm{NaBr}$ нанокристаллов фторида натрия $\mathrm{NaF}$ конусной формы. Именно образование кристаллов фторида натрия приводит к локальному изменению показателя преломления стекла $\left(\Delta n<10^{-3}\right)$ в облученной области по сравнению с необлученной областью $n \approx 1.49$. После термообработки для увеличения прозрачности записанный элемент можно отбелить, освещая зеленым лазерным излучением высокой мощности, соответственно линии поглощения серебра. Под действием импульсного лазерного излучения происходит фотофрагментация наночастиц серебра с образованием серебряных кластеров, а также фотоионизация нейтральных атомов серебра с переходом их в ионное состояние [7]. Этот эффект фотодеструкции может быть использован для создания прозрачных и высокоэффективных брэгговских дифракционных решеток, работающих в видимом диапазоне спектра.

\section{2. Схема записи}

В предложенном методе записи рельефно-фазовые дифракционные решетки применяются как фазовые маски для создания интерференционной картины в ближнем поле внутри фоточувствительной подложки, как схематично показано на рис. 1, $a$. Одиночный когерентный лазерный пучок освещает фазовую маску и формирует накладывающиеся пучки в дифракционных порядках, таким образом фазовая маска копируется в толстослойный голографический материал. Когерентная плоская волна падает на одномерную дифракционную решетку (фазовую маску) в плоскости, нормальной к штрихам решетки, непосредственно нанесенной на волновод [13]. Дифракция на неселективной рельефной решетке в качестве фазовой маски приводит к появлению трех пучков, которые распространяются внутри стеклянной подложки: проходящий (0-й порядок дифракции) и два дифрагированных (+1-й и -1 -й порядки). Поэтому в подложке формируются три области интерференции: область I соответствует интерференции +1-го и 0-го порядков дифракции, область II с интерференцией 0-го и -1-го порядков дифракции и область III с интерференцией всех трех +1-го, 0-го и -1 дифракционных порядков. На практике область III занимает большую часть объема записи из-за относительно небольшой толщины волновода $(\leq 2 \mathrm{~mm})$ по сравнению с апертурой записывающего пучка $(40 \mathrm{~mm})$ и состоит из трех периодичностей. Рабочая периодичность дифракционного волновода формируется интерференцией 0-го и -1-го порядков дифракции.

Записав фазовую маску один раз и выполняя затем процедуру копирования, мы поворачиваем только один компонент в записывающем пучке. Фоторезистивную рельефно-фазовую маску можно нанести непосредствен- 
$a$

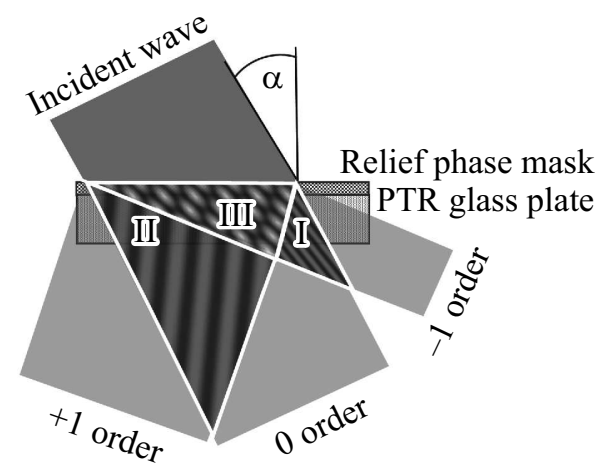

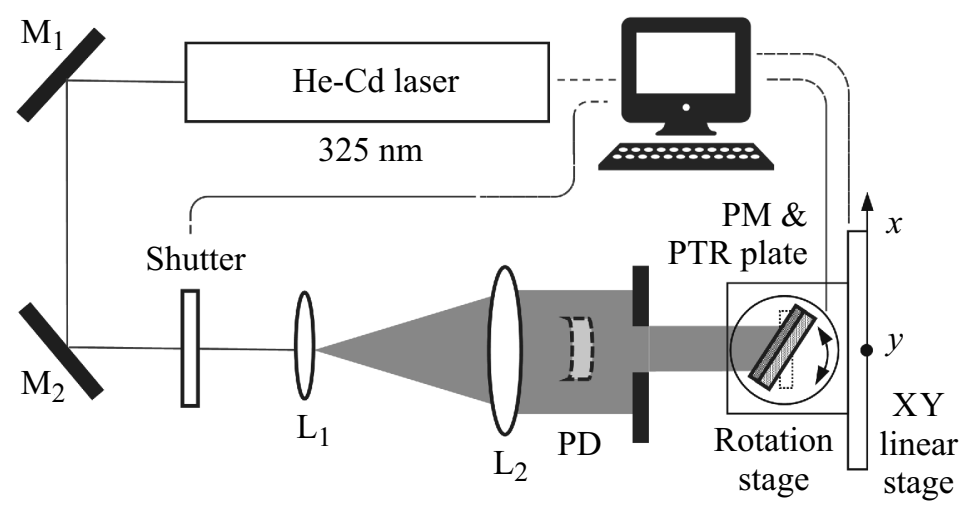

Рис. 1. (a) Схема записи экспериментальной установки. $\mathrm{M}$ - зеркало, L - линза, $\mathrm{PD}$ - фотодиодный измеритель мощности, PM - фазовая маска. (b) Схематичное изображение процесса записи в ФТР платине: I - область интерференции +1-го и 0-го порядков дифракции, II - область интерференции 0-го и -1-го порядков дифракции, III - область трехлучевой интерференции +1-го, 0-го и -1-го дифракционных порядков. Направление периодичности интерференционных полос от наложения 0-го и -1-го дифракционных порядков позволяет сформировать световодное направление.

но на ФТР пластину. ФТР стекло не фоточувствительно к излучению, к которому фоточувствителен фоторезист.

Схема стенда для получения волноводных брэгговских дифракционных решеток в ФТР стекле показана на pис. $1, b$. Пучок излучения гелий-кадмиевого лазера расширяется и коллимируется с помощью телескопической системы линз. „Сэндвич“ с фазовой маской (PМ) и ФТР подложкой установлен на моторизованном угловом позиционере. Коллимированный пучок под расчетным углом падения $\alpha$ направляется на ФТР-пластину (PTR plate), закрепленную на поворотном и линейном приводах, которые контролируют угол падения $\alpha$ и пространственное положение образца. Электромеханический затвор (Shutter) задает время экспонирования. Измеритель мощности (PD) временно устанавливается в направлении записывающего пучка для контроля экспозиции перед проведением записи. Процедура записи с необходимыми угловыми и планарными положениями осуществляется с помощью программного управления приводами в стенде записи.

\section{3. Мультиплексирование при записи через фазовую маску}

Брэгговские дифракционные элементы для дисплеев дополненной реальности состоят из нескольких мультиплексированных объемных решеток периодами $200-400 \mathrm{~nm}$. На рис. 1 показано одиночное экспонирование. Угловой шаг для мультиплексирования определяется угловой селективностью записанной брэгговской решетки и спектрально-угловым диапазоном светодиода при работе волновода.

Для анализа записываемой структуры проведем моделирование процесса интерференции. Пусть комплексные амплитуды интерферирующих волн имеют вид $\mathbf{E}_{1} \exp \left(-j \mathbf{k}_{1} \mathbf{r}\right), \mathbf{E}_{2} \exp \left(-j \mathbf{k}_{2} \mathbf{r}\right)$ и $\mathbf{E}_{3} \exp \left(-j \mathbf{k}_{3} \mathbf{r}\right)$, что соответствует трем порядкам дифракции, формируемым после фазовой маски. Тогда в результате интерференции этих волн в областях I, II, III получим распределение интенсивности света

$$
\begin{aligned}
& I(\mathbf{r})=\left|\mathbf{E}_{\Sigma}(\mathbf{r})\right|^{2}=\left|\sum_{n=1}^{3} \mathbf{E}_{n} \exp \left(-j \mathbf{k}_{n} \mathbf{r}\right)\right|^{2} \\
& =I_{0}\left[1+\sum_{i=1}^{2} \sum_{j=i+1}^{3} V_{i j} \cos \left(\mathbf{K}_{i j} \mathbf{r}+\varphi_{i j}\right)\right],
\end{aligned}
$$

где $I_{0}=\sum_{n=1}^{N}\left|\mathbf{E}_{n}\right|^{2}-$ величина полной интенсивности света, $V_{i j}=\left|\mathbf{E}_{i} \mathbf{E}_{j}^{*}\right| / I_{0}-$ контраст интерференционной полосы между $i$-м и $j$-м пучками, $\mathbf{K}_{i j}=\mathbf{k}_{i}-k_{j}-$ вектор решетки при интерференции между $i$-м и $j$-м пучками; волновые векторы $\mathbf{K}_{N}$ определяются по углам дифракции; $\varphi_{i j}=\operatorname{phase}\left[\mathbf{E}_{i} \mathbf{E}_{j}\right]-$ фаза интерференции между $i$-м и $j$-м пучками. Каждая пара интерферирующих пучков формирует одну синусоидальную решетку, охарактеризованную вектором $\mathbf{K}_{i j}$.

Моделирование в соответствии с формулой (1) продемонстрировано на рис. 2. Одиночная запись реализует структуру типа „поленница“, как показано на рис. 2, a. Формируется четыре направления интерференционных полос, определяемые углом падения оптического излучения на фазовую маску, лишь одно из которых представляет собой расчетное световодное направление: обеспечиваемое интерференцией 0-го и -1-го порядков дифракции. Угловое мультиплексирование, которое описано в данной работе, осуществляет поворот вектора решетки К. Рисунки $2, b$ и $c$ иллюстрируют результат пяти и десяти последовательных экспозиций фазовой маски соответственно.

Результаты моделирования показали, что даже при очень низкой дифракционной эффективности (в пределах $3 \%$ ) контраст интерференционных полос остается 
$a$

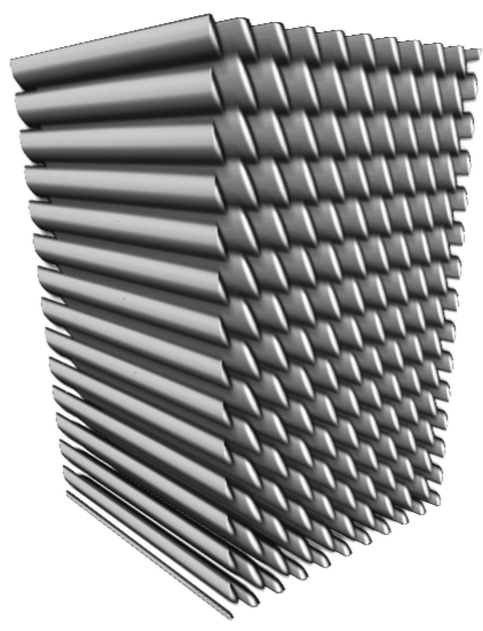

$b$

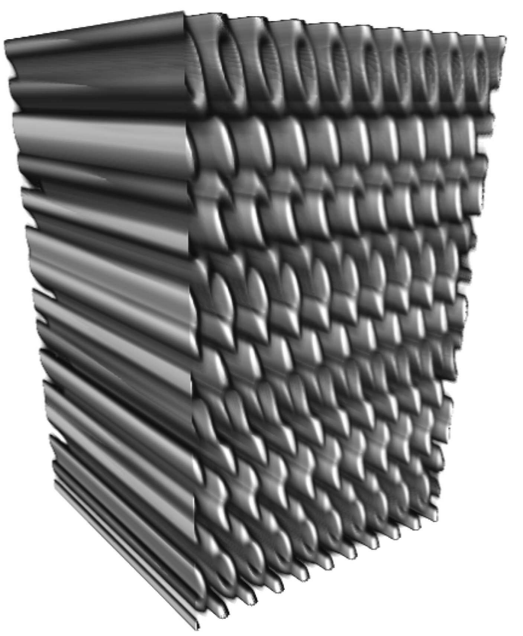

$c$

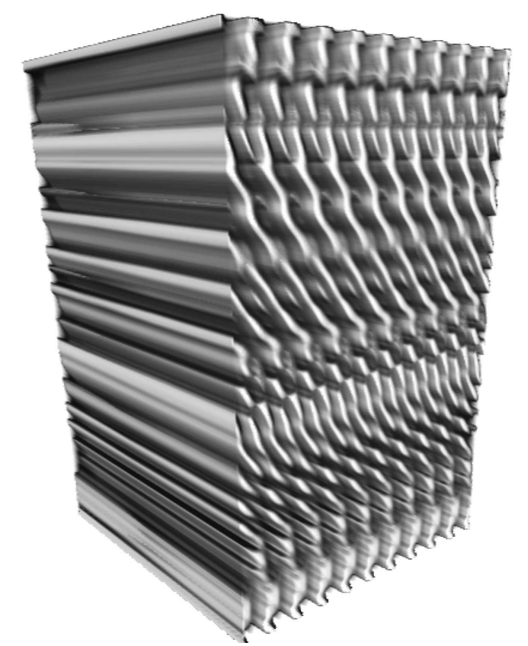

Рис. 2. 3D-модели записанных объемных структур с помощью рельефно-фазовой маски для однократной экспозиции $(a), b-$ для 5 последовательных экспозиций и $c$ - для 10 последовательных экспозиций.

выше 0.6 в области II при трехлучевой интерференции и выше 0.34 в областях I, III для двухлучевой интерференции (рис. 1, $a$ ). Необходимо отметить, что соответственно снижению контраста повышается минимальное значение величины интенсивности света в интерференционном поле. Этот факт необходимо учитывать при подборе экспозиции, чтобы полностью использовать динамический диапазон фоточувствительного материала.

\section{4. Расчет параметров записи}

Расчет самой фазовой маски основан на уравнении дифракции и базируется на периодах и углах наклона записываемой брэгговской решетки, которые необходимо реализовать. Скопированные брэгговские дифракционные решетки рассчитываем как волноводные голограммы для планарного ввода-вывода оптического излучения в стеклянных световодах [14]. Вводной Н1 и выводной Н2 элементы в пластине имеют одинаковую конфигурацию решеток, что можно интерпретировать как обратный ход работы выводного элемента по сравнению с вводным. Размер входного зрачка световода определяется размером генерируемого изображения для передачи в световод (выходного зрачка проектора). Входная Н1 и выходная Н2 голограммы имеют одинаковую конфигурацию для каждого ракурса в мультиплексе, т.е. одинаковые периоды и направления решетки $d_{\mathrm{H} 1}=d_{\mathrm{H} 2}$, $\gamma_{\mathrm{H} 1}=\gamma_{\mathrm{H} 2}$. Размер выходного зрачка световода зависит от возможностей выводных дифракционных решеток и определяет линейный размер изображения, наблюдаемого пользователем. Наряду с функцией вывода изображения Н2 расширяет выходной зрачок по одному из направлений. В работе рассматривается простейшая конфигурация волновода, в которой размножение зрачка происходит только в горизонтальном направлении.
Полагаем, что сгенерированное после осветителя изображение на входе в световод представляется набором плоских волн, падающих каждая под своим углом $\beta_{1}$ (в воздухе). Приходящий на каждую из голограмм пучок должен локально удовлетворять условию Брэгга для каждого мультиплекса из набора ракурсов. Правило знаков в текущем расчете: положительные значения углов отсчитываются влево относительно нормали, а отрицательные вправо. Угол распространения излучения в подложке $\beta_{2 n}$ ограничен условием ПВО $\beta_{\text {TIR }}$ для одного края поля и углом скольжения $\beta_{\text {slide }}$ для второго края поля. Причем для края поля, граниченного углом скольжения, важно, чтобы не было разрывов между зрачками при выводе изображения. Критический угол ПВО, при котором излучение „скользит“ вдоль границы подложки, равен $\beta_{\mathrm{TIR}}=\arcsin (1 / n)$. Для уверенного попадания в этот диапазон углов примем границы диапазона $\beta_{2 \text { left }}=\beta_{\text {slide }}-5^{\circ}, \beta_{2 \text { right }}=\beta_{\text {TIR }}+5^{\circ}$. Угловая селективность единичной брэгговской решетки с учетом ширины спектра светодиода соответствует значению $\Delta=2.4^{\circ}$ [6]. На угол записи $\alpha$ накладывается ограничение $\alpha_{1} \leq 45^{\circ}$ по технологичности наклонного освещения фазовой маски при копировании.

Период рельефно-фазовой маски $d_{s}$ рассчитывается таким образом, чтобы обеспечить нужные 0-й и -1-й порядки дифракции в УФ лазерного излучения. В среде должен существовать достаточный угол дифракции -1 -го порядка, который составляет $\alpha_{2 n} \leq 80^{\circ}$. Период фазовой маски, обеспечивающий эти возможности, определяется из уравнения решетки как

$$
d_{s}=-\lambda /\left(n \sin \left(\alpha_{2 n \max }\right)-\sin \left(\alpha_{1 \max }\right)\right) .
$$

Параметры решеток рассчитываются из условия Брэгга и однозначной косинусоидальной связи периода формируемой объемной структуры $d$ с периодом фазовой маски $d_{s}$, если правило знаков выбрано таким образом, 
что рассматривается -1-й порядок дифракции:

$$
\left\{\begin{array}{l}
\sin \left(\gamma-\beta_{1 n}\right)=-\frac{\lambda_{G}}{2 d n}=\sin \left(\frac{\beta_{1 n}+\beta_{2 n}}{2}-\beta_{1 n}\right)= \\
\sin \frac{\beta_{2 n}-\beta_{1 n}}{2} \\
d=d_{s} \cos \gamma=d_{s} \cos \frac{\beta_{1 n}+\beta_{2 n}}{2}=-\frac{\lambda_{G}}{2 n \sin \frac{\beta_{2 n}-\beta_{1 n}}{2}} \\
\beta_{2 n}=\arcsin \left(\sin \beta_{1 n}-\frac{\lambda_{G}}{n d_{s}}\right)
\end{array}\right.
$$

Таким образом, геометрия дифракции соответствует дифракции восстанавливающего излучения на рельефнофазовой маске. Затем рассчитывается соответствующая геометрия копирования (угол падения $\alpha$ ) для длины волны записи $\lambda=0.325 \mu$ m. Чтобы найти $\alpha_{1 n}$ и $\alpha_{2 n}$, решена система из двух уравнений, где одно является уравнением решетки для фазовой маски, а другое определяет угол наклона как половину угла между интерферирующими пучками (направлениями распространения 0-го и -1-го порядков дифракции):

$$
\left\{\begin{array}{l}
-\lambda_{U V}=d_{s} n\left(\sin \alpha_{2 n}-\sin \alpha_{1 n}\right) \\
\gamma=0.5\left(\alpha_{1 n}+\alpha_{2 n}\right)
\end{array}\right.
$$

Аналитическое решение относительно $\alpha_{1 n}$ имеет вид

$$
\begin{gathered}
\alpha_{1 n}=\gamma-\arcsin \frac{-\lambda_{U V}}{2 d_{s} n \cos \gamma}, \\
\alpha_{2 n}=2 \gamma-\alpha_{1 n} .
\end{gathered}
$$

Требуемое значение угла $\alpha$ записывающего луча составляет

$$
\alpha=\arcsin \left(n \cos \alpha_{1 n}\right) .
$$

В таблице показаны параметры записи, рассчитанные по формулам (2)-(5), которые были использованы в дальнейшем эксперименте для фазовой маски с периодом $419 \mathrm{~nm}$.

\section{2. Эксперимент}

\section{1. Условия записи и постобработки}

Стенд по голографическому копированию является виброустойчивым. Контроль уровня вибраций в эксперименте проводился с помощью микроинтерферометра в течение $1.25 \mathrm{~h}$. Измерения амплитуды вибраций (линейных смещений) проводились с использованием датчика перемещения Numerical Vision Displacement Meter и лазера Cobolt Flamenco DPSS (660 nm, $100 \mathrm{~mW})$. Время одного экспонирования с мультиплексной записью намного меньше, оно составляет 2 мин. Линейные смещения значительно превышают период записанных брэгговских дифракционных решеток, что подтверждает стабильность записи.

Этапы подготовки к записи включали нанесение фоторезиста на поверхность ФТР пластины, запись по расчетным параметрам и проявление фазовой маски, почернение задней поверхности и граней пластины. Чернение необходимо для устранения неблагоприятных переотражений при последующей голографической записи. После экспонирования ФТР стекла фоторезистивная фазовая маска удалялась с поверхности пластины. Постэкспозиционная обработка для ФТР стекла заключалась в высокотемпературной выдержке в электрической муфельной печи SNOL 7.2/1100L с керамической камерой в течение $10 \mathrm{~h}$ при температуре $\approx 500^{\circ} \mathrm{C}$. Для контроля температуры в процессе обработки был использован термометр Center 520. Завершающим этапом является обесцвечивание путем прямого сканирования лазерным пучком с использованием импульсного фемтосекундного мощного излучения зеленого лазера $(\lambda=515 \mathrm{~nm})$, что необходимо для повышения прозрачности изготавливаемой волноводной пластины.

\section{3. Экспозиционная характеристика}

При единичной или мультиплексной записи мы стремимся к равномерности дифракционной структуры и к максимизации дифракционной эффективности. Подбор режима экспозиции проводится из условия минимизации шума рассеяния и формирования баланса фоточувствительности.

На рис. 3 показаны экспозиционные характеристики для дифракционной эффективности (левая ось) и коэффициента пропускания (правая ось), полученные при регистрации единичных решеток через рельефнофазовую маску в ФТР пластине толщиной $2 \mathrm{~mm}$. Для записи использовался Не-Сd-лазер Kimmon IK3501R$\mathrm{G}$ мощностью $50 \mathrm{~mW}$ и длиной волны $325 \mathrm{~nm}$. Показанные на графиках значения экспозиции относятся к излучению, приходящему на фазовую маску, как показано для позиции $\mathrm{PD}$ на рис. $1, b$. После расширения пучка мощность излучения составляла около $2 \mathrm{~mW}$. За контраст при записи отвечает распределение энергии в дифракционных порядках, как упоминалось ранее.

Экспозиционные кривые для левой оси представляют зависимость дифракционной эффективности от экспозиции, для правой оси показана аналогичная зависимость для коэффициента пропускания, параметра, важного для дисплеев дополненной реальности. Особенностью волноводных дифракционных решеток является сложность прямых измерений дифракционной эффективности изза того, что первый дифракционный порядок „захвачен“ внутрь подложки. Поэтому исследование основано на косвенном контроле ослабления по измерению мощности проходящего луча. Измерения проводились с помощью зеленого лазера MSL-FN-532 (200 mW), измерителя мощности Ophir Photonics PD300 и поворотного позиционера Standa 8MR191 с угловым разрешением 4.5" с шагом 1/8. Показано, что рационально использовать дозы экспозиции $0.5-3 \mathrm{~J} / \mathrm{cm}^{2}$, на которых достигается дифракционная эффективность выше 50\%. При мощности записывающего пучка в представленном 
Рассчитанные параметры получения световодной пластины из ФТР-стекла

\begin{tabular}{|c|c|c|c|c|c|c|}
\hline \multirow[t]{2}{*}{ № } & \multirow{2}{*}{$\begin{array}{l}\text { Полевой угол } \\
\text { на входе } \beta_{1},{ }^{\circ}\end{array}$} & \multirow{2}{*}{$\begin{array}{c}\text { Угол наклона } \\
\text { страт } \gamma,{ }^{\circ}\end{array}$} & \multirow{2}{*}{$\begin{array}{c}\text { Период объемной } \\
\text { решетки } d, \mathrm{~nm}\end{array}$} & \multicolumn{2}{|c|}{ Углы при записи и после записи } & \multirow{2}{*}{$\begin{array}{c}\text { Угол копиро- } \\
\text { вания } \alpha,{ }^{\circ}\end{array}$} \\
\hline & & & & $\alpha_{1 n}, \circ$ & $\alpha_{2 n}, \circ$ & \\
\hline 1 & 10.8 & -18.72 & 397 & -3.14 & -34.31 & -4.78 \\
\hline 2 & 8.4 & -20.65 & 392 & -4.87 & -36.43 & -7.43 \\
\hline 3 & 6.0 & -22.63 & 387 & -6.63 & -38.63 & -10.13 \\
\hline 4 & 2.6 & -25.56 & 378 & -9.18 & -41.94 & -14.07 \\
\hline 5 & 1.2 & -26.81 & 374 & -10.25 & -43.38 & -15.73 \\
\hline 6 & -1.2 & -29.04 & 366 & -12.12 & -45.96 & -18.66 \\
\hline 7 & -2.6 & -30.39 & 361 & -13.23 & -47.55 & -20.42 \\
\hline 8 & -6.0 & -33.88 & 348 & -16.03 & -51.73 & -24.89 \\
\hline 9 & -8.4 & -36.61 & 336 & -18.13 & -55.09 & -28.30 \\
\hline 10 & -10.8 & -39.70 & 322 & -20.39 & -59.01 & -32.06 \\
\hline
\end{tabular}

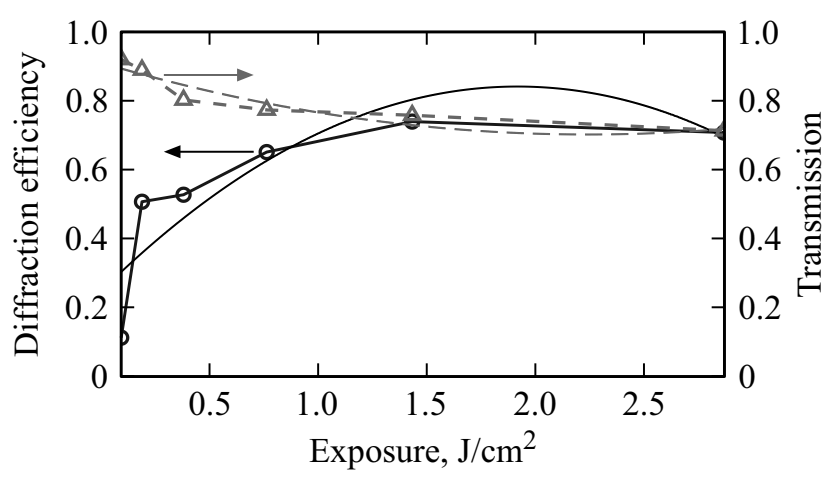

Рис. 3. Экспозиционные зависимости дифракционной эффективности (круги, сплошные линии) и пропускания (треугольники, штриховые линии) решеток, изготовленных с помощью рельефно-фазовой маски. Точки, соединенные ломаными линиями - результат измерений, гладкие линии - результаты аппроксимации.

эксперименте $2 \mathrm{mV}$, это соответствует времени экспонирования от 5 до $30 \mathrm{~h}$.

\section{1. Мультиплексирование}

В данном разделе описаны эксперименты по записи мультиплексных решеток с параметрами, представленными в таблице. На основе экспозиционной кривой (рис. 3), показывающей, при какой единичной экспозиции максимально эффективно используется модуляция показателя преломления ФТР стекла, мы выбирали продолжительность записи пропорционально количеству мультиплексов. Экспериментальная задача процедуры мультиплексирования - эффективно использовать модуляцию показателя преломления $\Delta n$, отвечающую за весь динамический диапазон [15]. Он распределяется в $N$-кратном мультиплексировании, что приводит к модуляции показателя преломления $n_{1}, n_{2}, \ldots, n_{N}$.

На рис. 4 показаны результаты эксперимента по увеличению количества мультиплексов при сохранении времени экспонирования $2 \mathrm{~h}$ для записи каждого ракурca, что допустимо в силу продолжительного линейного участка чувствительности ФТР стекла. Как и в случае с получением экспозиционной кривой, проводились косвенные измерения дифракционной эффективности по ослаблению прошедшего порядка.

В рамках эксперимента по 10-кратному мультиплексированию был выполнен анализ линейности фотоматериала, что продемонстрировано на рис. 5. Получили, что в пределах экспонирования единичного элемента от 40 до 90 s дифракционная эффективность полученных мультиплексных элементов варьируется незначительно.

Дифракционная эффективность отдельной решетки в $N$-кратном мультиплексе уменьшается примерно в $N$ раз, что подтверждается измерениями, показанными на рис. 6, a. Показано, как увеличение числа мультиплексов влияет на дифракционную эффективность при сохранении общего времени экспонирования. Соответствие количества мультиплексов графической характеристики указано в легенде к графику. Эксперименты показали, что для полученного волновода толщиной $2 \mathrm{~mm}$ можно было достичь эффективности дифракции $10-20 \%$ в каждом из 10 мультиплексов.

На рис. 6, $b$ показан один пучок зеленого лазерного излучения, отвечающий за одно угловое направление и распространяющийся посредством ПВО внутри изготовленного планарного волновода, описанного в разд. 1.3. Каждый мультиплекс обеспечивает свой собственный угол Брэгга, отвечающий за часть поля зрения в системе дополненной реальности. Входной дифракционный элемент (in-coupler) используется для согласования направлений распространения и дифракционной эффективности коллимированных пучков от проектора. В соответствии с контурами селективности дифракционного элемента световые пучки входят в волновод и затем распространяются в соответствующем направлении. Выходной элемент (out-coupler) работает в обратном ходе. Он расположен рядом с глазом наблюдателя и выводит излучение из подложки соответственно направлениям частей углового поля на входе. В исследуемом случае для входного элемента при отрицательных углах падения в воздухе создаются пропускающие мультиплексированные решетки, а для положительных углов падения в воздухе - отражательные. Для выходного элемента 

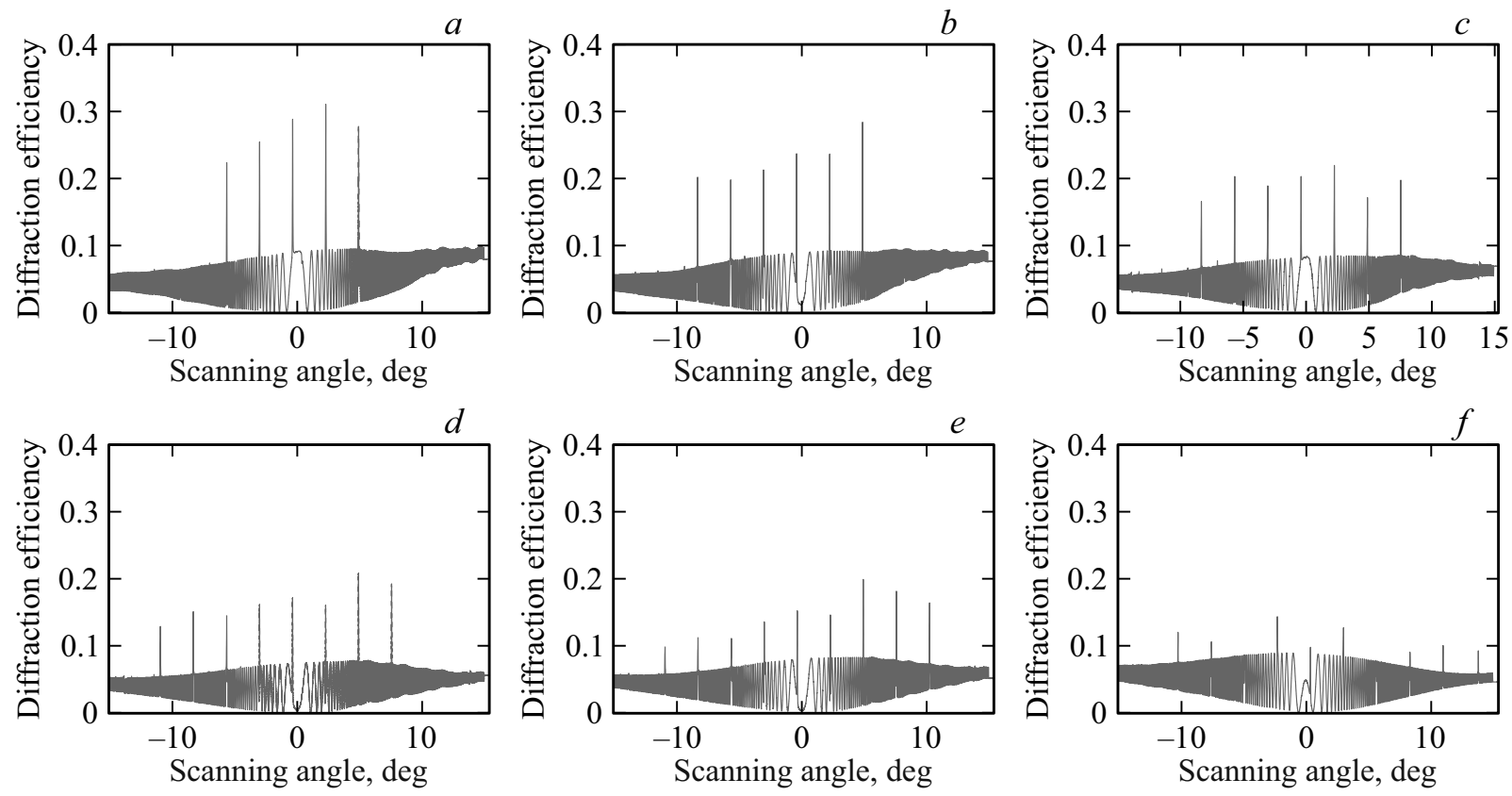

Рис. 4. Угловые характеристики дифракционной эффективности для мультиплексных брэгговских решеток, записанных с помощью рельефно-фазовой маски $a-5$-кратным, $b-6$-кратным, $c-7$-кратным, $d-8$-кратным, $e-9$-кратным и $f-10$-кратным мультиплексированием.
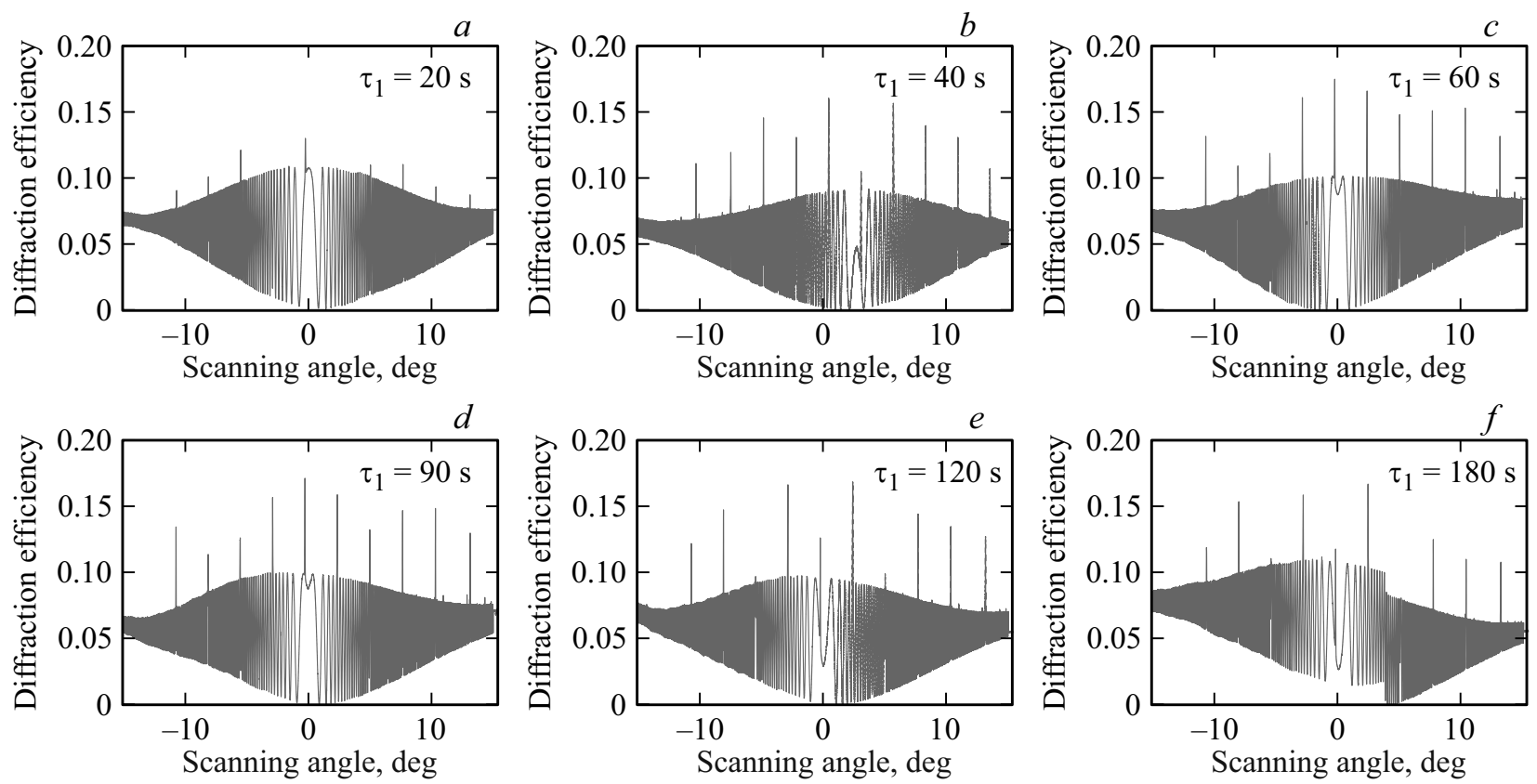

Рис. 5. Угловые характеристики дифракционной эффективности для 10 мультиплексных брэгговских решеток, записанных с помощью рельефно-фазовой маски при разном времени экспонирования одиночной голограммы: $a-20, b-40, c-60, d-90$, $e-120$ и $f-180 \mathrm{~s}$.

половина углового поля обеспечивается модами дифракции на отражение, а половина - модами дифракции на пропускание.

В описанном эксперименте для задачи ввода и вывода изображения оптимально по эффективности оказалось использовать голограммы с 7-кратным мультиплекси- рованием. Это зависит не только от свойств дифракционных элементов, но и от мощности излучения в модуле генерации изображения. На рис. 6,c показано выведенное для наблюдателя изображение, переданное с помощью плоскопараллельной пластины на рис. $5, b$, в которой дифракционные элементы ввода-вывода сформи- 

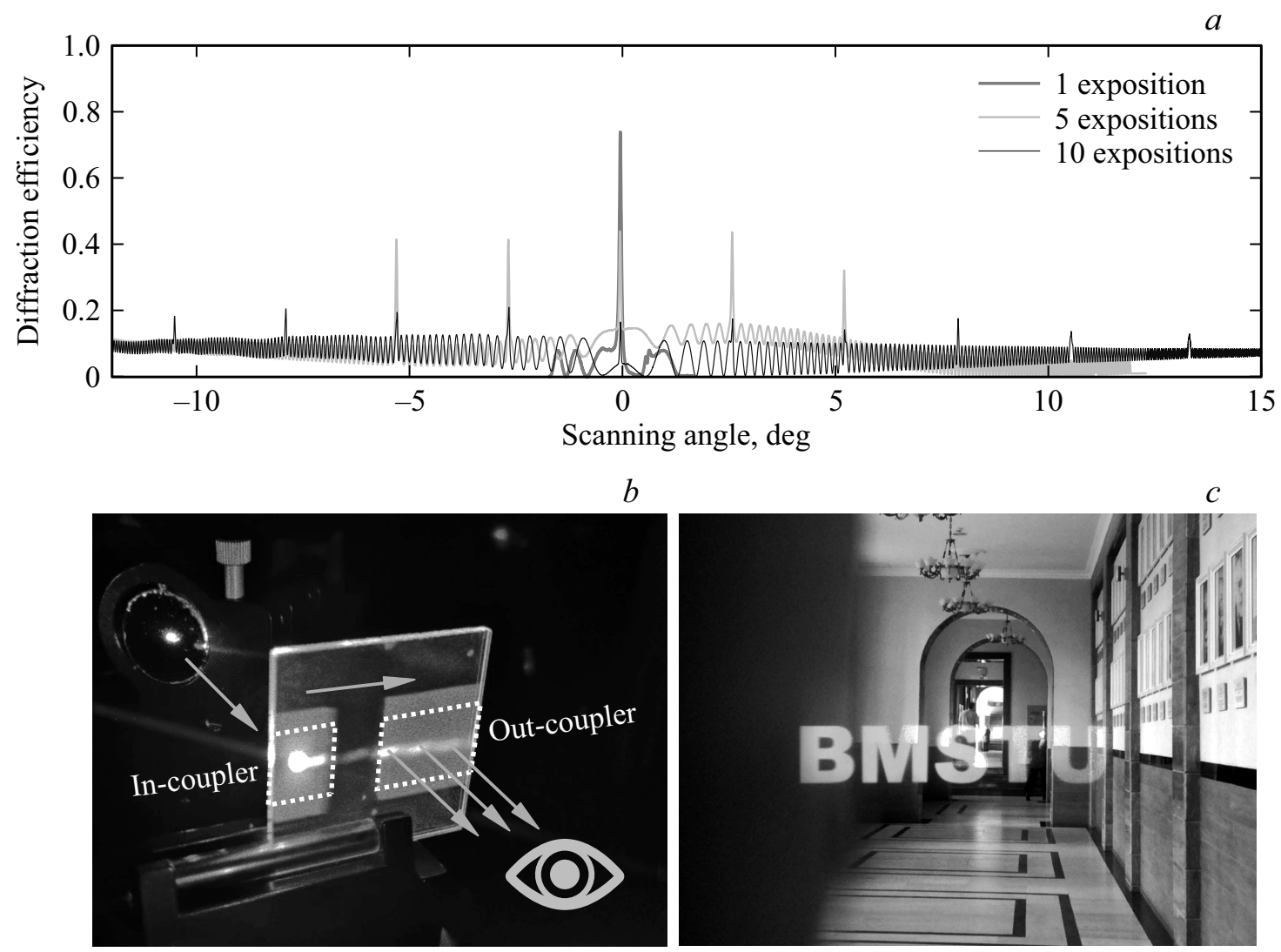

Рис. 6. (a) Угловая характеристика дифракционной эффективности для мультиплексных брэгговских решеток, записанных с помощью фазовой маски. $(b)$ Иллюстрация работы дифракционного волновода с одним введенным пучком внутри плоскопараллельной ФТР пластины. (c) Выводимое изображение на прозрачный дисплей на основе дифракционного волновода, в котором реализовано семикратное мультиплексирование.

рованы семью мультиплексами. Она была установлена в систему прозрачного дисплея с выводимым зеленым изображением от компактного проектора MiniRay c широким зеленым спектром.

\section{4. Заключение}

В представленном исследовании подход к записи основан на нескольких последовательных экспонированиях фазовой маски. Описан анализ процедуры мультиплексирования для сбалансированного согласования параметров угловой ширины полосы и дифракционной эффективности получаемых объемных дифракционных решеток. Угловое мультиплексирование (или оптическое копирование) помогает расширить эффективное угловое поле зрения в в окологлазных дисплеях дополненной реальности. Структурные параметры каждой мультиплексированной решетки оптимизированы в соответствии с направлениями распространения. Представленная процедура записи с мультиплексированием по углу более удобна при использовании неселективной рельефнофазовой маски. При стандартной записи интерференционной картины, когда реализован интерферометр с делением по амплитуде, сложно перенастраивать схему записи для широкого диапазона углов мультиплексирования. Но записав фазовую маску один раз и далее выполняя процедуру оптического копирования, мы вращаем только один компонент в регистрирующем пучке. При записи поверхностной фазовой маски в фоторезисте ее можно наносить непосредственно на ФТР пластину. Рельефно-фазовая маска способна обеспечить качественную мультиплексную запись, несмотря на неравенство дифракционной эффективности в порядках дифракции. Представленный метод позволяет сократить период регистрируемых оптических фазовых структур в 1.5 раза по сравнению с периодом фазовой маски и показывает высокий уровень повторяемости.

\section{Благодарности}

Авторы благодарят Н. Никонорова и С. Иванова за консультации по фото-термо-рефрактивному стеклу.

\section{Конфликт интересов}

Авторы заявляют, что у них нет конфликта интересов. 


\section{Список литературы}

[1] Zhang Y., Fang F. // Precision Engin. 2019. V. 60. P. 182-496.

[2] Lee Y.-H., Zhan T., Wu S.-T. // Virtual Reality \& Intelligent Hardware. 2019. V. 1. N 1. P. 10-20.

[3] Hsu F.-H., Han C.-Y., Chen K.-H., Hsu K.-Y., Chen J.-H. // Opt. Express. 2018. V. 26. N 16. P. 20534-20543.

[4] Yu C., Peng Y., Zhao Q., Li H., Liu X. // Appl. Opt. 2017. V. 56. N 34. P. $9390-9397$.

[5] Shen Z., Zhang Y., Liu A., Weng Y., Li X. // Opt. Mater. Express. 2020. V. 10. N 2. P. 312-322.

[6] Odinokov S.B., Shishova M.V., Markin V.V., Lushnikov D.S., Zherdev A.Y., Solomashenko A.B., Kuzmin D.V., Nikonorov N.V., Ivanov S.A. // Opt. Express. 2020. V. 28. N 12. P. 17581-17594.

[7] Nikonorov N., Ivanov S., Dubrovin V., Ignatiev A. // InTech. 2017. P. 435-461.

[8] Vanin V.A. // Soviet J. Quantum Electronics. 1978. V. 8. N 7. P. 809-818.

[9] Park T.-H., Kim S.-M., Oh M.-C. // Current Optics and Photonics. 2019. V. 3. N 5. P. 401-407.

[10] Weichelt T., Vogler U., Stuerzebecher L., Voelkel R., Zeitner U.D. // Opt. Express. 2014. V. 22. N 13. P. 16310 16321.

[11] Smith B.W. // J. Micro/Nanolith. MEMS MOEMS. 2019. V. 8. N 2. P. 021207.

[12] Shaoxin S., Ren X., Liu S., Yang Z., Zhang Y. // Opt. Eng. 2013. V. 52. N 9. P. 095103.

[13] Shishova M.V., Zherdev A.Y., Lushnikov D.S, Odinokov S.B. // Photonics. 2021. V. 7. Is. 4.

[14] Odinokov S.B., Zherdev A.Yu., Shishova M.V., Solomashenko A.B., Lushnikov D.S., Markin V.V. // Quantum Electronics. 2020. V. 50. N 7. P. 653-657.

[15] Zhai Q., Tao S., Zhang T., Song X., Wang D. // Opt. Express. 2009. V. 17. N 13. P. 10871-10880. 